\title{
CANCER V-ATPase EXPRESSION SIGNATURES: A DISTINCTIVE BALANCE OF SUBUNIT $C$ ISOFORMS IN ESOPHAGEAL CARCINOMA
}

Juliana do Couto Vieira Carvalho dos Santos ${ }^{1}$, Pedro Nicolau Neto², Evenilton Pessoa Costa ${ }^{1}$, Frederico Firme Figueira ${ }^{1}$, Tatiana de Almeida Simão ${ }^{3}$, Anna Lvovna Okorokova Façanha ${ }^{4}$, Luis Felipe Ribeiro Pinto ${ }^{2,3^{*}}$, Arnoldo Rocha Façanha $^{1^{*}}$

${ }^{1}$ Laboratório de Biologia Celular e Tecidual, Universidade Estadual do Norte Fluminense Darcy Ribeiro, Campos dos Goytacazes, RJ, Brazil

2Programa de Carcinogênese Molecular, Instituto Nacional de Câncer - INCA, Rio de Janeiro, RJ, Brazil

${ }^{3}$ Departamento de Bioquímica, Universidade Estadual do Rio de Janeiro, Rio de Janeiro, RJ, Brazil

${ }^{4}$ Laboratório de Fisiologia e Bioquímica de Microrganismos, Universidade Estadual do Norte Fluminense Darcy Ribeiro, Campos dos Goytacazes, RJ, Brazil

*Correspondence to: Arnoldo R. Façanha and Luis Felipe Ribeiro Pinto, emails: arnoldo.facanha@gmail.com; Ifpinto@inca.gov.br.

Keywords: ATP6V1C1, ATP6V1C2, isoform a, cancer biomarker, esophageal cancer, V-ATPase

\section{ABSTRACT}

V-ATPases are hetero-oligomeric enzymes consisting of 14 subunits and playing key roles in ion homeostasis and signaling. Differential expressions of these proton pumps have been implicated in carcinogenesis and metastasis. To elucidate putative molecular signatures underlying these phenomena, we evaluated the V-ATPase genes expression in Esophageal Squamous Cell Carcinoma (ESCC) using gene expression microarray data and extended the analysis to other cancers the Oncomine database. Among all differentially expressed genes, those encoding the V-ATPase $C$ isoforms exhibited striking expression patterns validated by qRT-PCR in paired ESCC samples and respective normal surrounding tissues. Structural modeling of $\mathrm{C} 2 \mathrm{a}$ isoform uncovered motifs for oncogenic kinases in an additional peptide stretch, and an actin-biding domain downstream to this sequence. This study reveals multicancer molecular signatures in the V-ATPase structure and establishes that the expression ratios of its subunits/isoforms could form a conformational code that controls the pump regulation and interactions related to tumorigenic events. 


\section{INTRODUCTION}

Esophageal cancer (EC), the sixth most common cause of death from cancer worldwide (Bray, Ferlay et al. 2018, Ferlay, Colombet et al. 2018), encompass two histological subtypes, esophageal adenocarcinoma (EAC) and esophageal squamous cell carcinoma (ESCC), the last one responsible for about $90 \%$ of all diagnosed cases (Rustgi and El-Serag 2014). The very poor overall survival of ESCC patients is mainly due to its late stage detection and poor response to treatments (Bird-Lieberman and Fitzgerald 2009, Roshandel, Nourouzi et al. 2013). Therefore, it is essential to understand the molecular mechanisms involved in ESCC towards identifying new therapeutic targets and molecular biomarkers that can improve the detection and clinical managements.

The V-type $\mathrm{H}^{+}$-ATPase (V-ATPase) has attracted increasing attention in the field of molecular oncology (Stransky, Cotter et al. 2016) due to its differential overexpression in tumor cell membranes (Sennoune, Bakunts et al. 2004, Capecci and Forgac 2013) and the consequent intra- and extracellular pH dysregulation (Webb, Chimenti et al. 2011), both associated with key tumorigenic cellular processes, such as, migration and invasion (Sennoune, Bakunts et al. 2004, Capecci and Forgac 2013, Cotter, Capecci et al. 2015), regulation of signaling pathways frequently altered in cancer (Lim, Park et al. 2007, Cruciat, Ohkawara et al. 2010, Zoncu, Bar-Peled et al. 2011, Sennoune and MartinezZaguilan 2012, Faronato, Nguyen et al. 2015), immunomodulation (Katara, Jaiswal et al. 2014, Ibrahim, Kulshrestha et al. 2016) and therapy resistance (Martinez-Zaguilan, Raghunand et al. 1999, De Milito and Fais 2005, Liao, Hu et al. 2007, Wojtkowiak, Verduzco et al. 2011, Lu, Lu et al. 2013). These ATPdependent proton pumps translocate $\mathrm{H}^{+}$ions across endomembranes of all eukaryotic cells and also through plasma membranes of some specialized cells, acidifying intracellular compartments and the extracellular matrix, respectively (Nishi and Forgac 2002, Forgac 2007, Cipriano, Wang et al. 2008). The VATPase is a complex enzyme composed of 14 subunits organized into a cytoplasmic domain (V1) responsible for ATP hydrolysis and a proton translocation transmembrane domain (V0. The V1 domain consists of eight (AH) subunits, while V0 sector, in human cells, is formed by the subunits $c, c$ ", $a, d$ 
and $e$, as well as two accessory subunits, Ac45 and M8-9 (Marshansky, Rubinstein et al. 2014, Cotter, Stransky et al. 2015).

The V-ATPase subunits $B, C, E, G, a, d$, and $e$ have multiple isoforms encoded by different genes, which have been postulated to exhibit tissue specific expression patterns (Smith, Borthwick et al. 2002, Sun-Wada, Yoshimizu et al. 2003, Toei, Saum et al. 2010). However, up to now, no clear functional roles have been attributed to the distinct isoforms, except for the four a isoforms described as responsible for the enzyme targeting to different cellular membranes (Kawasaki-Nishi, Bowers et al. 2001), by which a1 and a2 are found in pumps of intracellular membranes, while pumps assembled with a3 and a4 are usually directed to plasma membrane (Morel, Dedieu et al. 2003, Toyomura, Murata et al. 2003, Wagner, Finberg et al. 2004, Toei, Saum et al. 2010, Capecci and Forgac 2013). In addition, it has also been demonstrated that yeast V-ATPase complexes containing two different $a$ isoforms show distinct cellular localization, coupling efficiency and regulation by association/dissociation of the V1 and V0 domains (Kawasaki-Nishi, Nishi et al. 2001, Samarao, Teodoro et al. 2009). VATPase plays essential roles in a variety of cellular processes, and the existence of multiple subunit isoforms might reflect multifunctional conformations, including specific compositions for specific cell types and pathophysiological conditions. Here, we investigated the differential expression patterns of the V-ATPase subunits/isoforms in human ESCC and in other types of tumors, exploring the potential of these proton pumps as putative cancer biomarkers for early diagnosis and more effective prognosis.

\section{RESULTS}

\section{Expression profile of V-ATPase subunits in ESCC}

The expression profiles of 25 genes encoding V-ATPase subunits were obtained from an ESCC transcriptome developed by the Brazilian National Institute of Cancer - INCA (Nicolau-Neto, Da Costa et al. 2018). An unsupervised hierarchical clustering analysis showed that ESCC exhibits striking changes in expression of most isoforms of the V-ATPase subunits in comparison with normal esophageal tissues excised surrounding the tumors, in a profile which clearly 
segregates these two groups and suggests a pattern of tumor-specific V-ATPase expression (Figure. 1a; the fold change and $p$-value of each gene is shown in Table S1). The genes were considered differentially expressed when their $p$ value was less than 0.005 . It is of note that the $A$ and $B$ subunits, which constitute the evolutionary conserved catalytic domain of the V-ATPase, presented no significant expression changes (ATP6V1A: 1-fold change and $\mathrm{p}=0.78$; ATP6V1B1: 1 -fold change and $\mathrm{p}=0.79$; ATP6V1B2: -1.08 -fold change and $\mathrm{p}=$ 0.45) (Table 1, Figure S1).

The subunit $c$ " (ATP6VOB), which compose the proteolipid ring of the VATPase, exhibited higher expression in ESCC samples than in normal tissues. Even more interesting were the changes found among the isoforms expression of the same subunit, as observed for $d$ and G subunits, where, $d 2$ (ATP6V0D2) and G2 (ATP6V1G2) isoforms were more expressed in tumor, while $d 1, G 1$ and G3 isoforms (ATP6V0D1, ATP6V1G1 and ATP6V1G3, respectively) were less expressed in tumor compared with normal surrounding tissue (Figure S2).

However, among all differentially expressed V-ATPase genes, only genes for the subunits $C$ and a presented the highest differences among their respective isoforms. In ESCC compared with normal surrounding tissue, ATP6V1C1 and TCIRG1 mRNAs were upregulated (2.79 and 1.53-fold change, respectively), while those of ATP6V1C2 and ATP6VOA4 were downregulated (-1.28 and -2.76fold change, respectively). In addition, significant differences in expression in these four genes were also observed between ESCC and healthy esophageal tissues from individuals without cancer (Figure $\mathbf{1 b}$ and $\mathbf{c}$; Figure S3). The TCIRG1 and ATP6VOA4 genes encode two of the four distinct $a$ isoforms, a3 and a4 (V0 sector), whereas in the $\mathrm{V}_{1}$ domain, ATP6V1C1 and ATP6V1C2 encode the different $C$ subunit isoforms; ATP6V1C1 encodes the $C 1$ isoform (NCBI gene ID: 528) and ATP6V1C2 encodes two alternative transcriptional splice variants, namely C2a and C2b isoforms (NCBI gene ID: 245973).

To investigate the expression pattern of V-ATPase genes in others cancer types, we systematically analyzed mRNA transcriptome between normal tissues and tumor tissues of 18 sites comprising different histological types using the Oncomine database. The threshold was designated according to the 


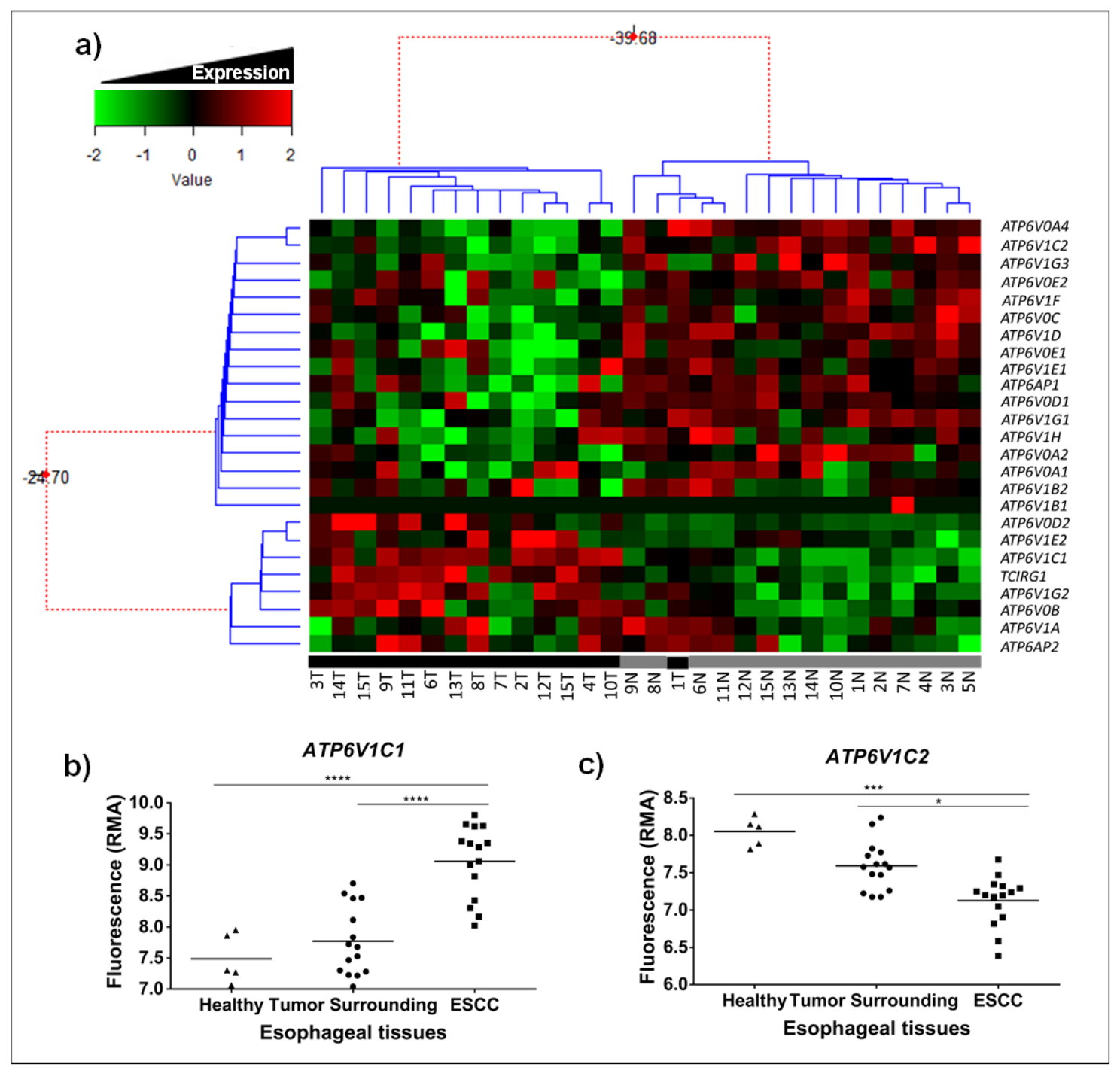

Figure 1. V-ATPase expression profile in ESCC by microarray database. (a)

Unsupervised hierarchical clustering analysis of 15 esophageal samples (tumors and respective surrounding tissues) showing the expression of the 25 genes of V-ATPase. Green indicates decreased gene expression and red increased genes expression. $\mathrm{N}$ and black bar = normal surrounding tissue; $\mathrm{T}$ and grey bar $=$ Tumor. (b) ATP6V1C1 and ATP6V1C2 expression levels in tumor, normal-appearing surrounding tissue and healthy esophageal mucosa from individuals without cancer. ${ }^{*} p<0.05$; ${ }^{* * *} p<0.001$; ${ }^{* * *} p<0.0001$.

following values: $p$-value $1 \mathrm{E}-4$, fold-change 2 , and top gene ranks $10 \%$. Was possible to verify a common regulation of the V-ATPase isoforms expression in different tumor types. Remarkably, ATP6V1C1 and TCIRG1 expression was constantly higher in most cancer types compared to normal tissues, while ATP6V1C2 and ATP6VOA4 were downregulated, in the same way as founding 
the ESCC microarray experiments (Figure S4). These results imply a coordinate expression and a balance between specific isoforms of the subunits $C$ and $a$ in tumors.

\section{ATP6V1C1 and ATP6V1C2 transcripts are differentially expressed in ESCC}

In order to validate the most expressive results of the microarray analysis, we evaluated ATP6V1C1 and ATP6V1C2 mRNA levels by RT-PCR in 38 ESCC paired samples (tumors and respective normal surrounding tissues). The ATP6V1C1 expression in ESCC relative to respective normal tissue ranged from 0.43 to 7.37 -fold change, with the median value of 2.33-fold change. Assuming a fold change cut-off of $\geq 2$, about half $(54 \%)$ of the ESCC samples presented ATP6V1C1 mRNA levels increased when compared to their respective normal surrounding tissues (Figure 2a), while the expression of the ATP6V1C2 gene was downregulated in most of the tumors analyzed, considering a cut-off $\leq-2$ (67.6\% for v1; 72.2\% for v1,2). For ATP6V1C2 fold change values ranged from 0.46 to -48.9 with the median value of -3.96 for $v 1$ and of -0.51 to -52.9 with the median value of -6.53 for $v 1,2$ (Figure $\mathbf{2 b}$ and $\mathbf{c}$ ).

Next, a comparative analysis of mRNA levels of subunit $C$ isoforms in tumors and normal surrounding tissues was performed. Expression levels of ATP6V1C1 in ESCC samples group was approximately 2.5-fold higher than those detected in the normal surrounding tissues group (median values of 0.008196 and 0.003301, respectively) (Figure 2d). For ATP6V1C2-v1 and ATP6V1C2-v1,2 the mRNA levels were significantly lower in the ESCC group than those in the normal surrounding tissues group, with the median values of -4.3 and -9.6 -fold, respectively ( $v 1$ : median of 0.000489 in ESCC and 0.00241074 normal surrounding; $v 1,2$ : median of in 0.000629 ESCC and 0.006044 normal surrounding) (Figure. 2e and 2f). These findings are in agreement with the data obtained from microarray analysis (Figure 1), confirming that the V-ATPase $C$ isoforms present a remarkable differential expression in ESCC, when compared to normal surrounding tissue. 


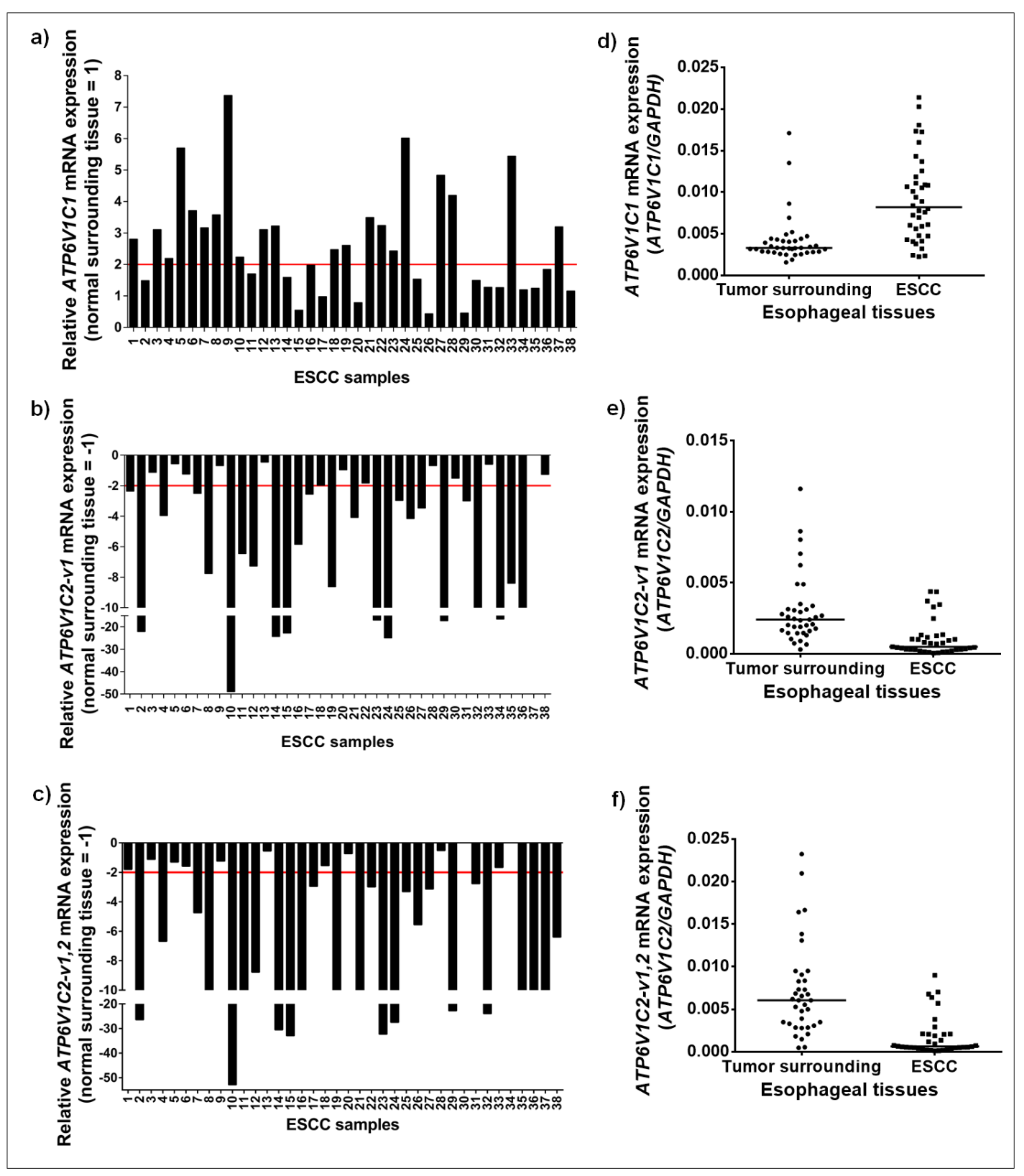

Figure 2. The expression pattern of V-ATPase $C$ isoforms in ESCC. Left images: RT-PCR analysis of (a) ATP6V1C1, (b) ATP6V1C2-v1 and (c) ATP6V1C2-v1,2 mRNA levels in paired ESCC samples. ESCC samples presenting over 2-fold increase (red dashed line) in relative ATP6V1C1 expression were considered upregulated and ESCC samples presenting decrease of -2-fold (red dashed line) in relative ATP6V1C2 expression were considered downregulated. Values are expressed as relative to those obtained in tumors respective normal surrounding tissue (= 1 or -1$)$. Right images: qRT-PCR evaluation of (d) ATP6V1C1, (e) ATP6V1C2-v1 and (f) ATP6V1C2-v1, 2 mRNA levels distribution in the groups of normal surrounding and their paired ESCC tissues. Values are shown in relative units. mRNA levels were normalized by those of $G A P D H$, used as the housekeeping gene. $p<0.0001$. 
We further examined the expression ratio of ATP6V1C1 and ATP6V1C2 isoforms (C1/C2-v1 and $C 1 / C 2-v 1,2$ ratios). The median value of $C 1 / C 2-v 1$ ratio was of 1.4-fold in normal surrounding tissues and 13.4-fold in the ESCC samples, while the median value of $C 1 / C 2-v 1,2$ ratio was of 0.5 -fold and 10.1 -fold in the normal surrounding and ESCC tissues, respectively (Figure S5). These results suggest that the expression levels of the different $C$ isoforms are carefully guarded to achieve a proportional balance in normal tissues, which dysregulation towards the $C 1$ prevalence over the $C 2$ is an intrinsic characteristic of ESCC.

\section{C isoforms as potential diagnostic biomarker for ESCC}

In order to evaluate whether $C$ isoforms mRNA expression could be used to discriminate between tumor and non-tumor esophageal tissues, we performed the Receiver Operating Characteristic (ROC) curve analysis using the gene expression values from ESCC and normal surrounding tissues. The differential expression of ATP6V1C1 and ATP6V1C2 was able to accurately discriminate ESCC samples from normal surrounding tissues $(\mathrm{p}<0.0001)$, with sensitivity and specificity of $79.49 \%$ and $84.62 \%$ for ATP6V1C1; $83.78 \%$ and $83.78 \%$ for ATP6V1C2-V1 and $80.56 \%$ and $86.11 \%$ for ATP6V1C2-V1,2, respectively. The ROC curve analysis for the $C 1 / C 2$ expression ratios generated an area under curve greater than 0.94 , reflecting the high accuracy of the test to discriminate tumors and normal surrounding tissues. The $C 1 / C 2-v 1$ and $C 1 / C 2-v 1,2$ ratios showed respectively a sensitivity of $86.49 \%$ and $88.89 \%$ and a specificity of $91.89 \%$ and $91.67 \%$ (Figure 3 ). These results reveal that mRNA expression ratio of the subunit $C$ isoforms can be useful as a diagnostic biomarker for ESCC.

\section{Expression profiles of V-ATPase genes in EC histological subtypes}

The expression profile of two genes of the V-ATPase subunit C in ESCC prompted us to investigate if such dysregulation is involved in esophageal carcinogenesis in general, independently of histological subtype. To this end, we used TCGA database to analyze the expression levels of V-ATPase genes in ESCC and EAC. The expression profile of all V-ATPase genes presented a very similar pattern in both histological subtypes (Figure 4a). Regarding to $C$ isoforms, both EAC and ESCC tumors exhibited higher expression levels of ATP6V1C1 than ATP6V1C2. The ATP6V1C1 expression was increased 6.6-fold when 
compared to ATP6V1C2 expression in EAC tissues. In ESCC samples, ATP6V1C1 was 11.6-fold more expressed than ATP6V1C2, corroborating with the data obtained by qRT-PCR, where ATP6V1C1 was 12.7 -fold more expressed in tumors than ATP6V1C2 (Figure 4b). Finally, the ATP6V1C1 expression levels were similar between the two-histological subtypes (expression median of 1578 for ESCC and 1479 for EAC), however, the average expression of ATP6V1C2 was lower in ESCC than in EAC (expression median of 136 for ESCC and 225 for EAC) (Figure S6).

a)

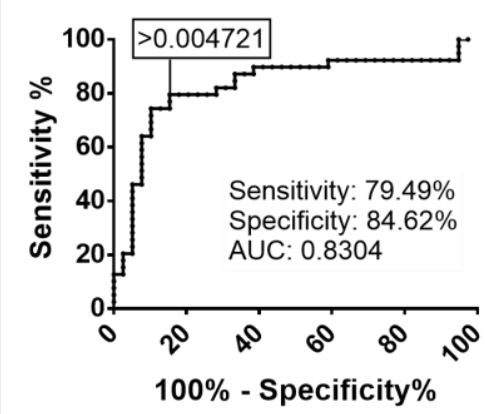

d)

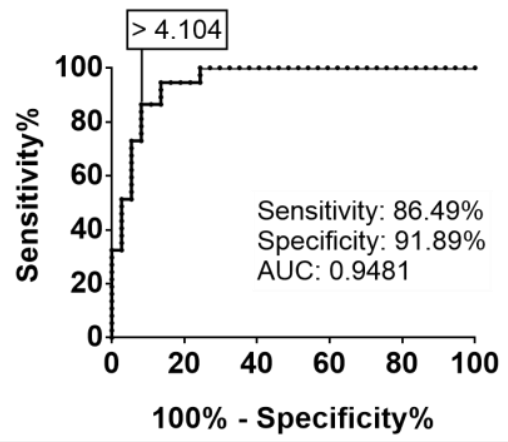

b)

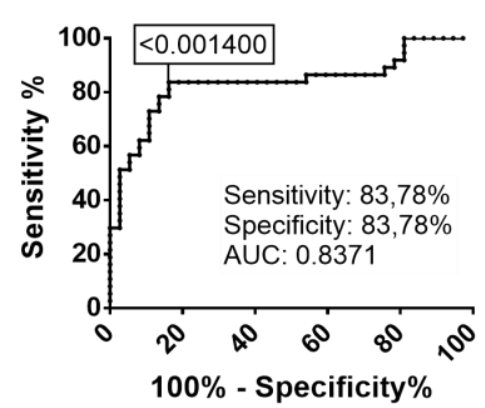

c)

\section{ATP6V1C2-v1,2}

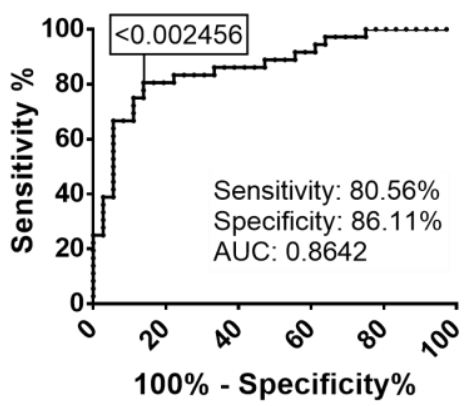

C1/C2-v 1,2 ratio

e)

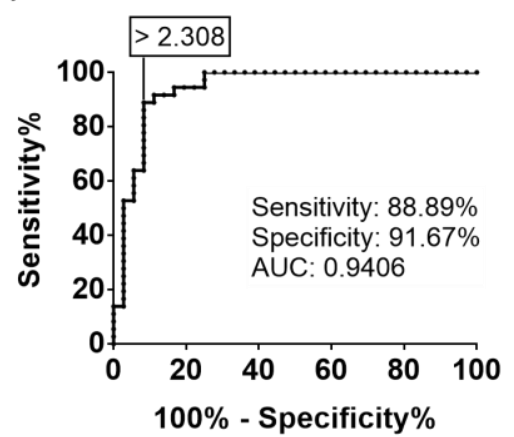

Figure 3. ROC curves of $C$ isoforms mRNA expression for the discrimination of ESCC tissue from normal surrounding mucosa samples. $\mathrm{ROC}$ curves are relative to mRNA expression of: (a) ATP6V1C1, (b) ATP6V1C2-v1, (c) ATP6V1C2-v1,2, (d) C1/C2-v1 ratio and (e) C1/C2-v1,2 ratio. The area under curve (AUC) indicates the accuracy of the test in discriminating tumors from normal surrounding tissues. Numbers in a box correspond to a cut-off used for the determination of the sensitivity and specificity. ROC, receiver operating characteristic. $p<0.0001$. 


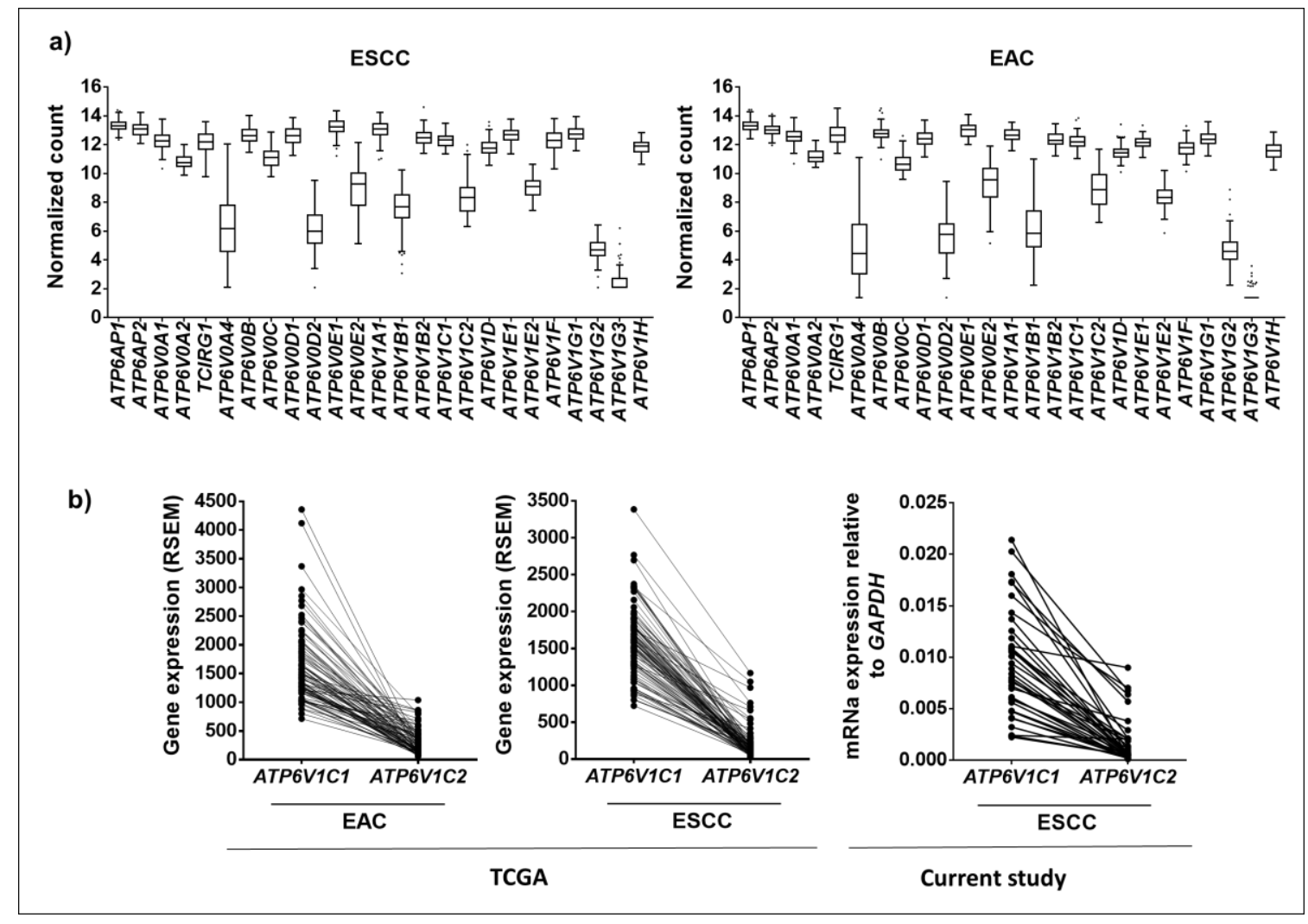

Figure 4. V-ATPase mRNA expression in esophageal cancer histological subtypes. (a) Expression profile of all V-ATPase genes in esophageal adenocarcinoma (EAC) and ESCC. (b) Respective ATP6V1C1 and ATP6V1C2 mRNA levels in EAC and ESCC assessed by RNA-sequencing data from TCGA and by $q R T-P C R$ in the current study.

\section{Structural modeling of the subunit $\mathrm{C}$ isoforms}

In addition, we performed the analysis of conserved domains among the human V-ATPase subunits C1 (NP_001686.1) (hC1), C2a (NP_001034451.1) (hC2a) and C2b (NP_653184.2) (hC2b) using the program BLASTP 2.8.0 in the mammalian taxon of the database NCBI Protein Reference Sequences. The three amino acid (aa) sequences were found to be highly conserved, and hC1 (382 aa), hC2a (427 aa) and hC2b (381 aa) share at least 55,3\% identity and $\sim 74,2 \%$ similarity (Table 2 ). When compared to isoform hC2b, the isoform hC2a has an additional exon that encodes for 46 aa at the position 276-321 aa. The BlastP analysis suggested that the isoform $\mathrm{hC2} 2 \mathrm{a}$ is present in all mammals, but absent in other taxa (yeast, nematode, mollusk, arthropod, birds, reptiles, amphibia and higher plants). Next, were performed analysis for protein-protein binding and for phosphorylation sites, exclusively on hC2a additional exon. These 


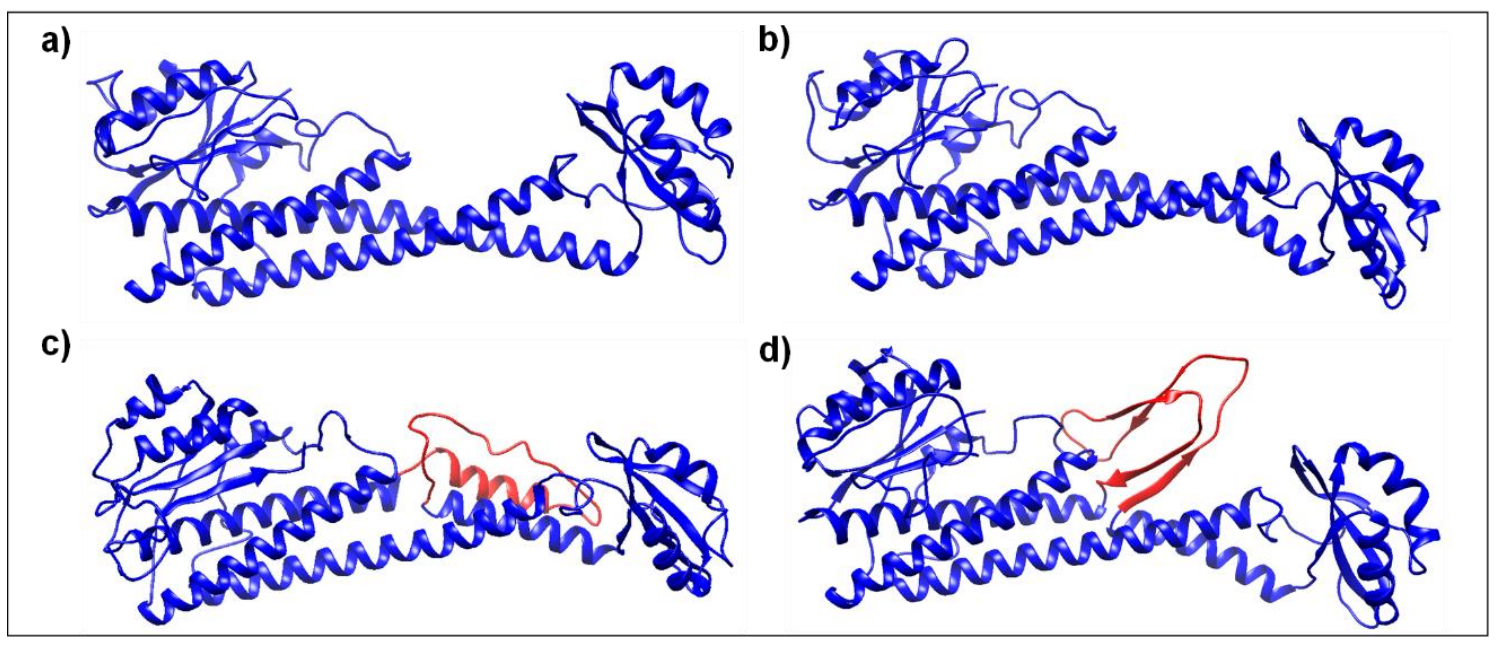

Figure 5. Prediction of three-dimensional structure of $C$ subunit isoforms. (a) hC1 isoform, (b) hC2b isoform, (c) most promising model of hC2a isoform and (d) model of $\mathrm{hC} 2 \mathrm{a}$ isoform validated by protein docking. $\mathrm{C} 1$ and $\mathrm{C} 2 \mathrm{~b}$ were modeled by homology using as template the resolved structure from yeast V-ATPase (3J9V.pdb) and C2a was obtained by ab initio approach. The additional peptide stretch present in the hC2a isoform is shown in red.

analyses revealed interesting motifs for serine/threonine kinases and phosphatases, such as ERK1 and 2, Casein Kinase II, PKA, PKC; and three potential binding motifs for proteins which recognize phosphorylated serinebased motifs with binding domains to WW, MDC1 BRCT and Plk1 PBD (Tables 3 and 4). No tyrosine kinase/phosphatase binding sites were identified in this specific region. Predictions of $\mathrm{hC} 1, \mathrm{hC} 2 \mathrm{a}$ and $\mathrm{hC} 2 \mathrm{~b}$ three-dimensional structures were performed by homology modeling and ab initio approaches (Figure 5). The RMSD assessment also indicated that $\mathrm{hC} 1$ and $\mathrm{hC} 2 \mathrm{~b}$ are structurally very similar, while hC2a has an additional structural motif (Table 2). Analysis of global and local stereochemical quality using validation programs revealed that the most stable structure predicted for the additional exon of hC2a would be an alpha-helix conformation flanked by unstructured regions (Figure 5c). However, since the subunit $C$ binds to, and serves as a substrate for PKA and PKC (Voss, Vitavska et al. 2007), these proteins were also used to refine the validation of the hC2a structural model. Following a series of protein-protein dockings between proposed hC2a models with PKA and PKC structures (Figure S7), the conformational model that best established PKA/PKC-hC2a interaction complexes was the $\mathrm{hC} 2 \mathrm{a}$ predicted model for which the domain corresponding to 
the additional exon is in a beta-sheet flanked by unstructured regions (Figure 5d, Figure S7).

\section{DISCUSSION}

In recent years, there has been an increasing body of evidence establishing that the V-ATPase proton pump is implicated in several cancer hallmarks (Hanahan and Weinberg 2011), such as, resistance to cell death, increased cell proliferation, invasion and metastasis, induction of angiogenesis, immune system modulation and reprogramming of energy metabolism (Webb, Chimenti et al. 2011, Stransky, Cotter et al. 2016, Pamarthy, Kulshrestha et al. 2018). However, despite the large amount of data accumulated on V-ATPase differential expression in various types of tumors, no clear molecular signature could be related to the carcinogenesis process in general. In previous reports, the overexpression of different subunits and isoforms have been highlighted in different cancers (Whitton, Okamoto et al. 2018), including a3 isoform in ovarian cancer (Kulshrestha, Katara et al. 2015), C1 isoform in oral cancer (Otero-Rey, Somoza-Martin et al. 2008), G1 isoform in glioblastoma (Di Cristofori, Ferrero et al. 2015), c subunit in hepatocellular carcinoma (Xu, Xie et al. 2012), and E1 isoform in EC (Son, Kim et al. 2016). This study aims at shedding a new light on this controversial issue by looking at the gene expression pattern for all V-ATPase subunits in ESCC and analyzing the expression balances among the putatively isoforms distinctive in relation to normal tissues. The data obtained not only open a new perspective on the structural and functional bases of the differential activation for this proton pump in ESCC, but also provide compelling evidences for wider significance to the carcinogenesis process in general. For this purpose, we have also performed the Oncomine database analysis, which revealed that specific expression patterns of V-ATPase subunits are not restricted to esophageal cancers since a similar imbalance of isoforms found in ESCC was also detected in different cancer types (Figure S4). The differential expression pattern we have found in tumors, encompassing all V-ATPase subunits isoforms, might also imply in specific coupling regulation and activation patterns quite different from that exhibited by normal cells, but highly functional for the cancer specific ion signaling and energy transduction demands. 
Our findings also suggest that the ratio of subunits isoforms can be part of a cellular code that controls the regulation of V-ATPase and tumorigenic related events. The data highlighted isoforms of subunits $a, C, d, G$, and the subunit $c "$ as the most differentially expressed in ESCC (Figure S2), which in turn are subunits involved in the structural and functional coupling between the domains V1 and V0. This reinforces the idea that the active V-ATPase holoenzyme conformations in tumors and normal tissues might assume quite distinct regulatory couplings. Perhaps not surprisingly, subunits $A(A T P 6 V 1 A)$ and $B$ (ATP6V1B1 and ATP6V1B2), which integrate the ATP hydrolysis catalytic domain, displayed no significant differential expression in ESCC comparing to normal esophageal tissues (Figure S1), suggesting a conserved and stable expression of the main subunits required for the substrate hydrolysis. Accordingly, the V-ATPase catalytic domain has been found to be highly conserved not only during molecular evolution and speciation, but also in relation to the counterpart domain of the F-ATPase synthase (Cross and Muller 2004).

We observed the most significant changes in the subunits $a$ and $C$, which presented the highest differential expression among their respective isoforms not only in ESCC (Figure 1, Figure S3) but also in other cancers as revealed by the Oncomine analysis (Figure S4). Our data, in conjunction with other information in the literature, have revealed tumor-specific imbalances in the expression of these subunits. For instance, in our analyzes the isoforms a3 (TCIRG1) and a4 (ATP6V0A4) were upregulated and downregulated, respectively, in tumors as compared to normal tissues. Likewise, in ovarian cancer tissues, the a3 isoform was found to be conspicuously expressed compared to normal samples (Kulshrestha, Katara et al. 2015). Overexpression of a3 isoform results in the targeting of V-ATPases to the plasma membrane of breast cancer cells, and confers high invasiveness to breast cancer and melanoma cells lines (Nishisho, Hata et al. 2011, Capecci and Forgac 2013). Moreover, a knockdown of a4 did not affect invasion of breast cancer cells, but unexpectedly resulted in a 2-fold increase in the a3 mRNA levels (Capecci and Forgac 2013), which is in line with our results suggesting a tightly regulated balance among the isoforms of these V-ATPase subunits. 
Our approach can also help to clarify the functional and evolutionary rational for the existence of such isoforms, an issue that remains poorly understood, since previous works have generally addressed only the most expressed isoforms for each subunit. This is the case of the C1 isoform (ATP6V1C1), for which the overexpression (Otero-Rey, Somoza-Martin et al. 2008) and related potential as biomarker (Perez-Sayans, Reboiras-Lopez et al. 2010) have been described in oral cancer. The $C 1$ isoform overexpression has also been correlated with rearrangements of actin filaments in breast cancer cells (Cai, Liu et al. 2014), and its silencing prevented breast cancer growth and bone metastasis in murine model (Feng, Zhu et al. 2013). More recently, it was reported that ATP6V1C1 enhanced breast cancer growth through activation of the mTORC1 pathway (McConnell, Feng et al. 2017). On the other hand, the C2a and C2b isoforms (ATP6V1C2) have been much less studied, most likely because these isoforms were initially proposed to be expressed specifically in kidney, lung and epididymis (Smith, Borthwick et al. 2002, Sun-Wada, Murata et al. 2003, Sun-Wada, Yoshimizu et al. 2003). However, we have found similar levels of expression for both $C 1$ and $C 2$ isoforms in normal esophageal surrounding tissues, contrasting with their expression imbalance in tumors characterized by a shift towards a strong $C 1$ dominance (Figure 2 and Figure S5).

The validation of the transcriptome data by $q R T-P C R$ reinforced that the ESCC group exhibits an increase of about 2.5-fold in ATP6V1C1 mRNA expression and a decrease of almost 10-fold in ATP6V1C2 expression compared with the normal surrounding tissues group. Furthermore, we demonstrated the potential of these isoforms to be used as diagnostic markers, since their mRNA levels were able to clearly discriminate ESCC from normal surrounding esophageal tissues, with the best results achieved using the $C 1 / C 2$ ratio (Figure 2 and 3) rather than any individual expression pattern. This highlights an interconnectivity among the $C$ isoforms expression, with a wide range of ATP6V1C1 and ATP6V1C2 expression between the tumors of different individuals, but invariably involving a quite proportional prevalence of the ATP6V1C1 expression over that of ATP6V1C2. The analysis of TCGA database confirmed that the imbalance between the ATP6V1C1 and ATP6V1C2 genes expression towards the prevalence of the first over the second is a clear 
molecular signature of esophageal tumors, regardless the histological type (Figure 4). It has been shown that EAC shares several molecular alterations with gastric adenocarcinoma, whilst ESCC is closer related to squamous carcinomas of head and neck (Nancarrow, Clouston et al. 2011). In fact, EAC and ESCC differ in histological type, areas of incidence around the world, risk factors and characteristic molecular alterations (Bird-Lieberman and Fitzgerald 2009, Rustgi and El-Serag 2014). Therefore, the similar expression patterns for $C$ isoforms observed in both tumors provides further evidence for this distinctive imbalance of $C$ isoforms as a molecular signature of the proton pump in different cancers.

This finding is also of special interest since subunit $C$ contributes to the structural and regulatory assembly of V-ATPase through the reversible dissociation of V1 and V0 domains (Puopolo, Sczekan et al. 1992, Curtis, Francis et al. 2002, Smardon and Kane 2007). Interestingly, the $C$ subunit is the only of the 14 subunits that is released into the cytosol during the regulatory disassembly of the proton pump (Kane and Parra 2000). In insect models, which have no alternative isoform for $C$ subunit in their genomes, this single subunit binds to polymeric F-actin and monomeric G-actin and increases the initial rate of actin polymerization in a concentration-dependent manner, regulating the thickness of actin filaments cross-linking (Vitavska, Merzendorfer et al. 2005). The interaction of actin and $C$ subunit might also contribute to stabilize the proton pump in its assembled state (Serra-Peinado, Sicart et al. 2016). Likewise, the cytoskeleton rearrangement somehow interconnected with the V-ATPase activity has been implicated in key cancer-related processes, such as increased migration and invasion (Cai, Liu et al. 2014), release of exosomes containing DNA, mRNA, miRNA and protein of tumors (Liegeois, Benedetto et al. 2006), membrane fusion (Strasser, Iwaszkiewicz et al. 2011), and receptors recycling (Nishi and Forgac 2002). Therefore, it seems likely that humans and other complex organisms that express multiple $\mathrm{C}$ isoforms would exhibit a wider array of actin-proton pump interactions and enzymatic regulation, in comparison to other organisms encoding only one type of subunit $\mathrm{C}$.

To shed light on this issue, we modelled the three-dimensional structures of each $C$ isoform in silico. The most striking difference between $\mathrm{C} 1, \mathrm{C} 2 \mathrm{~b}$ and $\mathrm{C} 2 \mathrm{a}$ isoforms concerns an additional stretch of 46 amino acids endowed with binding 
sites for different kinases and phosphatases (Figure 5, Tables 3 and 4), suggesting post-translational modifications in C2a-assembled holoenzyme. A structural model for C2a isoform, in which the additional peptide stretch assumed a beta sheet folding flanked by unstructured regions was produced by SwissModel (Holliday 2014). Conversely, stereochemical quality analysis performed in this study highlighted an alpha-helix bend flanked by unstructured regions as the most stable structural model for such region (Figure 5c). To clarify this question, we simulated a docking between the predicted models for C2a and PKA and PKC kinases that are known to bind to the subunit $C$ (Voss, Vitavska et al. 2007). The only model that allows C2a binding to PKA and PKC was that with the additional peptide stretch structured in a beta sheet folding flanked by unstructured regions (Figure 5d, Figure S7). It is possible that both conformations can be expressed, each one favored by different metabolic situations, especially considering that the $C$ subunit is exposed to distinct microenvironments, in the membrane bound holoenzyme and free in the cytosol during the dissociation $\mathrm{V} 1-\mathrm{V} 0$ regulatory cycle. This mechanism also involves the interaction of the $C$ and $B$ subunits with actin filaments (Serra-Peinado, Sicart et al. 2016), and based on the actin-binding domain described for the $B$ subunit (Holliday, Lu et al. 2000, Chen, Bubb et al. 2004, Zuo, Vergara et al. 2008), we found similar domains in the $C$ subunit isoforms (Figure S8). Curiously, the sequence corresponding to the putative actin binding site in the C2a isoform was located immediately downstream the additional peptide stretch, found only in this isoform and predicted to be highly mobile, what seems quite mechanistically sound.

Nonetheless, the mRNA of ATP6V1C2-v2 (C2b isoform) was found to be barely expressed in the evaluated samples, precluding its relative quantification by qPCR. It was also possible to determine a highly significant correlation between mRNA levels of ATP6V1C2-v1 and ATP6V1C2-v1,2 ( $r=0.9521$, $p<0.00001)$. Therefore, it is likely that that most of the expression observed in ATP6V1C2-v1,2 analysis is due to the expression of the variant 1 (C2a isoform). In this context, the observed switch towards $C 1$ in tumors assumes a major importance, as the additional peptide stretch in the C2a isoform may integrate kinases-dependent and actin binding mechanisms and other related regulatory functions of normal cells, which are disturbed as the $C 1$ isoform becomes 
ubiquitously prevalent in cancer cells. This hypothesis suggested by our in silico models should prompt future studies on the functional relevance of this C2a specific peptide stretch for the $\mathrm{H}^{+}$-pump regulatory coupling and cytoskeleton interactions.

In conclusion, to the best of our knowledge, this is the first report on multicancer molecular signatures for the V-ATPase holoenzyme, indicating a key role for the differential expression of their subunits and isoforms to form a conformational code, which ultimately would lead to specific $\mathrm{pH}$ signals and energy transductions related to carcinogenesis. Compelling evidence was also provided for a clear distinctive imbalance of the $C$ subunit isoforms pointing to ATP6V1C1 and ATP6V1C2 as promising targets for development of new cancer biomarkers and further in-depth mechanistic characterization. Taken together these findings open a new avenue towards understanding the functions of the differential expression of V-ATPase subunits isoforms, which hopefully would lead to future breakthroughs in exploration of this proton pump mechanistic role in carcinogenesis, cancer early diagnosis and therapeutic potential.

\section{MATERIALS AND METHODS}

\section{V-ATPase gene expression in ESCC Transcriptome}

V-ATPase gene expression profile of ESCC data were analyzed using transcriptome data developed by the Brazilian National Institute of Cancer - INCA (Nicolau-Neto, Da Costa et al. 2018). Microarrays were performed using a genechip Human Exon 1.0 ST array (Affymetrix, Inc.) and compared 15 ESCC paired samples (ESCC and respective surrounding tissues) and 5 healthy esophageal tissues of individuals without cancer. The raw data were normalized in Expression Console software (Affymetrix) using robust multi-array average (RMA) method. The differential expression was analyzed using the "Limma" package of $\mathrm{R}$ software (http://www.bioconductor.org/packages/release/bioc/html/limma.html) (Diboun, Wernisch et al. 2006, Ritchie, Phipson et al. 2015).

\section{V-ATPase gene expression by Oncomine database}


The expression levels of V-ATPase genes in 18 cancer types were obtained from the Oncomine database (https://www.oncomine.org/resource/login.html) (Rhodes, Yu et al. 2004). The fold-change of mRNA expression in cancer tissue compared to in their normal tissues was acquired using parameters of a threshold $p$-value of $1 \mathrm{E}-4$; foldchange of 2; and gene ranking in the top $10 \%$.

\section{Patients and samples}

Thirty-eight patients enrolled from 2008 to 2015 at the Brazilian National Cancer Institute (INCA) who had confirmed ESCC diagnosis and had not undergone chemotherapy and/or radiotherapy were recruited. Paired biopsies (tumors and normal-appearing surrounding mucosa, collected at least $5 \mathrm{~cm}$ far from the tumor border) were included in real-time PCR analysis. Epidemiological and clinicopathological data were obtained through interviews by using a standardized questionnaire and from patient's medical records, including whenever possible sex, age at diagnosis, tobacco smoking, alcohol intake, tumor stage and differentiation, esophageal localization and overall survival time after diagnosis. Individuals were classified regarding tobacco smoking as smokers (for ever- or ex-smoking) or never-smoking and regarding alcohol intake as drinkers (for ever- or ex-drinkers) or never-drinkers alcohol intake. The use of the human samples was approved by the Ethic Committee of the institution (INCA - 116/11). All patients, who kindly agreed to participate in the study, signed an informed consent form prior to study enrollment

\section{Clinicopathological features}

The clinicopathological characteristics of the 38 ESCC patients evaluated in the study are presented in Table 5. The median age of patients was 57 years, ranging from 48 to 79 years, male patients represented $84.2 \%$ of cases and near $90 \%$ of all patients were alcohol consumers and/or smokers. Tumors were most often located in the middle third of the esophagus (47.4\%), with a high prevalence of advanced stage (III or IV) of the disease (48\%) and poorly or moderately differentiated $(97.4 \%)$ culminating in a high mortality rate $(81.6 \%)$ with median overall survival of 7.73 months. 


\section{Real-time PCR}

Total RNA was extracted from the fresh tumor and normal surrounding esophageal tissues using RNeasy mini kit (Qiagen), according to the manufacturer's protocol. RNA concentration and purity were determined by spectrophotometry, and $500 \mathrm{ng}$ of total RNA was used for reverse transcription in a final volume of $20 \mu \mathrm{L}$ with SuperScriptTM II Reverse Transcriptase (Invitrogen $\AA$ ), following the manufacturer's protocol. Expression levels were detected by StepOnePlus ${ }^{\text {TM }}$ Real-Time PCR System (Applied) and each reaction $(12 \mu \mathrm{L})$ contained the corresponding cDNA (1:10), $900 \mathrm{ng}$ of each primer and 6.0 $\mu \mathrm{L}$ of Power SYBR Master Mix PCR (Applied). The reaction conditions were as follows: $95^{\circ} \mathrm{C}$ for $10 \mathrm{~min} ; 95^{\circ} \mathrm{C}$ for $15 \mathrm{sec}, 60^{\circ} \mathrm{C}$ for $1 \mathrm{~min}$, for $40 \mathrm{cycles}$; at the end a melting curve analysis was included, and fluorescence was measured from 60 to $99^{\circ} \mathrm{C}$ (each sample was analyzed in duplicate). Relative mRNA levels were quantified using the comparative cycle threshold method $(\triangle \triangle \mathrm{Ct})$ and normalized by the GAPDH expression and using the normal surrounding tissue as the reference $\left(2^{-\triangle \triangle C t}\right.$ formula). The expression ratio between ATP6V1C1 and ATP6V1C2 isoform C transcripts was calculated for each patient.

The oligonucleotide sequences are shown in the Table 6. Transcripts of the ATP6V1C2 variant 2 could not be evaluated individually due to detection limit of real-time PCR equipment that was not able to determine quantitatively with accuracy its very low RNA concentrations $\left(\mathrm{C}_{T}>32\right)$. Therefore, the transcripts of ATP6V1C1, ATP6V1C2 variant 1 (v1) and ATP6V1C2 variants 1 and $2(\mathrm{v} 1,2)$ were evaluated together. The cDNA samples availability determined the respective number of assays, 38 paired samples for the ATP6V1C1, 37 for ATP6V1C2-V1 and 36 for ATP6V1C2-V1,2.

\section{Analysis of ATP6V1C1 and ATP6V1C2 expression from data deposited in The Cancer Genome Atlas (TCGA) database}

Gene expression data from EC samples $(n=182)$, both esophageal adenocarcinoma $(n=87)$ and squamous cell carcinoma $(n=95)$, were downloaded from the public database cBioPortal for Cancer Genomics (Cerami, Gao et al. 2012, Gao, Aksoy et al. 2013), which provides visualization, analysis, and 
download of largescale data sets deposited in The Cancer Genome Atlas (TCGA).

\section{In silico 3D models of $C$ subunit isoforms, specific peptide domains, and protein-protein interaction sites and docking assays}

Conserved polypeptide sequences were estimated by using the $\mathrm{NCBi}$ database and the program BLASTP 2.8.0 (Boratyn, Schaffer et al. 2012), while the analysis of conserved domains was verified by using the CDD/SPARCLE (Marchler-Bauer, Bo et al. 2017). The identification of sites for phosphorylation/dephosphorylation and protein-protein interactions was performed using the Human Protein Reference Database (Amanchy, Periaswamy et al. 2007). The prediction of 3D models for hC1 (NP_001686.1), hC2b (NP_653184.2) and hC2a (NP_001034451.1) isoforms was performed by homology and ab initio modeling by four distinct programs: Modeller 9.19 (Sali and Blundell 1993), Swiss-Model (Biasini, Bienert et al. 2014), RaptorX (Kallberg, Wang et al. 2012) and I-Tasser (Yang, Yan et al. 2015). Analysis of global and local stereochemical quality for all predicted models were also performed by using different programs: Rampage (Lovell, Davis et al. 2003), Verify3D (Eisenberg, Luthy et al. 1997), ProSA (Wiederstein and Sippl 2007), VoroMQA (Olechnovic and Venclovas 2017), ProQ3D (Uziela, Menendez Hurtado et al. 2017), Qprob (Cao and Cheng 2016), DeepQA (Cao, Bhattacharya et al. 2016) and SVMQA (Manavalan and Lee 2017). The best models were refined using the ModRefiner program (Xu and Zhang 2011). Protein-protein docking assays were carried out using the programs ClusProV2 (Comeau, Gatchell et al. 2004) and HADDOCK (van Zundert, Rodrigues et al. 2016). In order to select the best interactions and to discard false positives, extensive analysis were carried out with the programs: DockScore (Malhotra, Mathew et al. 2015), PPCheck (Sukhwal and Sowdhamini 2015) and CCharPPI (Moal, Jimenez-Garcia et al. 2015). After selection of the protein complexes with native interaction characteristics, the prediction of binding free energy $(\Delta G-\mathrm{kcal} / \mathrm{mol})$ and the dissociation constant $\left(K_{\mathrm{d}}\right)$ of the complexes were calculated using the program PRODIGY (Vangone and Bonvin 2015, Xue, Rodrigues et al. 2016). Native complexes and their interactions were modeled by the program UCSF Chimera 
1.11.2 (Pettersen, Goddard et al. 2004). Multiple sequence alignments were generated using ClustalW (http://www.genome.jp/tools-bin/clustalw).

\section{Statistical Analysis}

The data were statistically analyzed using GraphPad Prism 5 Software (San Diego, CA, USA) and the difference considered significant at a $p$ value of $<0.05$. Unpaired $t$ test or Mann-Whitney test was applied when comparing two groups. When comparing three or more groups, one-way ANOVA or Kruskal-Wallis test and Dunn's posttest were used. A receiver operating characteristic ( $\mathrm{ROC})$ curve was plotted to investigate whether $C$ isoforms expression could be used as a marker to discriminate ESCC from normal-appearing surrounding mucosa.

\section{ACKNOWLEDGEMENTS}

We thank all patients involved in this study, the staffs of Endoscopy Service and National Tumor Bank of the Brazilian National Cancer Institute for their contribution in sample collection.

\section{ADDITIONAL INFORMATION}

\section{Competing interests}

The authors have no conflicts of interest.

\section{Funding}

This work was supported by grants from Conselho Nacional de Desenvolvimento Científico e Tecnológico (CNPq) and Fundação de Amparo à Pesquisa do Estado do Rio de Janeiro (FAPERJ, Brazil). This study was financed in part by the Coordenação de Aperfeiçoamento de Pessoal de Nível Superior - Brasil (CAPES) - Finance Code 001 (PhD fellowship to JCVCS and Post-doctoral fellowship to EPC). JCVCS received Post-doctoral fellowship from Universidade Estadual do Norte Fluminense Darcy Ribeiro (UENF) and FFF received a PhD fellowship from FAPERJ. The funders had no role in study design, data collection and analysis, decision to publish, or preparation of the manuscript.

\section{Author contributions}


JCVCS performed most of the experiments and analysis. PNN participated in the analysis and acquisition of data. EPC performed the in silico structural models. ARF and LFRP coordinated the project. JCVCS and ARF wrote the manuscript. JCVCS, PNN, TAS, ARF and LFRP performed study design. TAS and PNN participated in the collection of samples. ALOF and FFF provided specialized scientific and technical support. All authors discussed the results and manuscript text. All authors read and approved the final manuscript.

\section{Ethics}

Human subjects: The use of the human samples was approved by the Ethic Committee of the institution (INCA - 116/11). All patients, who kindly agreed to participate in the study, signed an informed consent form prior to study enrollment.

\section{Author ORCIDS}

Juliana C V C Santos https://orcid.org/0000-0001-5858-6081

Pedro Nicolau https://orcid.org/0000-0003-3322-2120

Evenilton P Costa https://orcid.org/0000-0003-3316-2834

Frederico F Figueira https://orcid.org/0000-0003-1366-0035

Tatiana A Simão https://orcid.org/0000-0001-8509-2247

Anna L O Façanha https://orcid.org/0000-0002-6856-7711

Luis F R Pinto https://orcid.org/0000-0001-5509-1008

Arnoldo R Façanha https://orcid.org/0000-0002-5641-6852

\section{Abbreviations}

DAB: Diaminobenzidin; EAC: Esophageal adenocarcinoma; EC: Esophageal cancer; ESCC: Esophageal squamous cell carcinoma; GAPDH: Glyceraldehyde3-phosphate dehydrogenase; TCGA: The Cancer Genome Atlas. 


\section{REFERENCES}

Amanchy, R., B. Periaswamy, S. Mathivanan, R. Reddy, S. G. Tattikota and A. Pandey (2007). "A curated compendium of phosphorylation motifs." Nat Biotechnol 25(3): 285-286.

Biasini, M., S. Bienert, A. Waterhouse, K. Arnold, G. Studer, T. Schmidt, F. Kiefer, T. Gallo Cassarino, M. Bertoni, L. Bordoli and T. Schwede (2014). "SWISS-MODEL: modelling protein tertiary and quaternary structure using evolutionary information." Nucleic Acids Res 42(Web Server issue): W252-258.

Bird-Lieberman, E. L. and R. C. Fitzgerald (2009). "Early diagnosis of oesophageal cancer." $\mathrm{Br} \mathrm{J}$ Cancer 101(1): 1-6.

Boratyn, G. M., A. A. Schaffer, R. Agarwala, S. F. Altschul, D. J. Lipman and T. L. Madden (2012). "Domain enhanced lookup time accelerated BLAST." Biol Direct 7: 12.

Bray, F., J. Ferlay, I. Soerjomataram, R. L. Siegel, L. A. Torre and A. Jemal (2018). "Global cancer statistics 2018: GLOBOCAN estimates of incidence and mortality worldwide for 36 cancers in 185 countries." CA Cancer J Clin 68(6): 394-424.

Cai, M., P. Liu, L. Wei, J. Wang, J. Qi, S. Feng and L. Deng (2014). "Atp6v1c1 may regulate filament actin arrangement in breast cancer cells." PLoS One 9(1): e84833.

Cao, R., D. Bhattacharya, J. Hou and J. Cheng (2016). "DeepQA: improving the estimation of single protein model quality with deep belief networks." BMC Bioinformatics 17(1): 495.

Cao, R. and J. Cheng (2016). "Protein single-model quality assessment by feature-based probability density functions." Sci Rep 6: 23990.

Capecci, J. and M. Forgac (2013). "The function of vacuolar ATPase (V-ATPase) a subunit isoforms in invasiveness of MCF10a and MCF10CA1a human breast cancer cells." J Biol Chem 288(45): 32731-32741.

Cerami, E., J. Gao, U. Dogrusoz, B. E. Gross, S. O. Sumer, B. A. Aksoy, A. Jacobsen, C. J. Byrne, M. L. Heuer, E. Larsson, Y. Antipin, B. Reva, A. P. Goldberg, C. Sander and N. Schultz (2012). "The cBio cancer genomics portal: an open platform for exploring multidimensional cancer genomics data." Cancer Discov 2(5): 401-404.

Chen, S. H., M. R. Bubb, E. G. Yarmola, J. Zuo, J. Jiang, B. S. Lee, M. Lu, S. L. Gluck, I. R. Hurst and L. S. Holliday (2004). "Vacuolar H+-ATPase binding to microfilaments: regulation in response to phosphatidylinositol 3-kinase activity and detailed characterization of the actin-binding site in subunit B." J Biol Chem 279(9): 7988-7998.

Cipriano, D. J., Y. Wang, S. Bond, A. Hinton, K. C. Jefferies, J. Qi and M. Forgac (2008). "Structure and regulation of the vacuolar ATPases." Biochim Biophys Acta 1777(7-8): 599-604.

Comeau, S. R., D. W. Gatchell, S. Vajda and C. J. Camacho (2004). "ClusPro: an automated docking and discrimination method for the prediction of protein complexes." Bioinformatics 20(1): 4550 .

Cotter, K., J. Capecci, S. Sennoune, M. Huss, M. Maier, R. Martinez-Zaguilan and M. Forgac (2015). "Activity of plasma membrane V-ATPases is critical for the invasion of MDA-MB231 breast cancer cells." J Biol Chem 290(6): 3680-3692.

Cotter, K., L. Stransky, C. McGuire and M. Forgac (2015). "Recent Insights into the Structure, Regulation, and Function of the V-ATPases." Trends Biochem Sci 40(10): 611-622.

Cross, R. L. and V. Muller (2004). "The evolution of A-, F-, and V-type ATP synthases and ATPases: reversals in function and changes in the H+/ATP coupling ratio." FEBS Lett 576(1-2): 1-4.

Cruciat, C. M., B. Ohkawara, S. P. Acebron, E. Karaulanov, C. Reinhard, D. Ingelfinger, M. Boutros and C. Niehrs (2010). "Requirement of prorenin receptor and vacuolar H+-ATPase-mediated acidification for Wnt signaling." Science 327(5964): 459-463.

Curtis, K. K., S. A. Francis, Y. Oluwatosin and P. M. Kane (2002). "Mutational analysis of the subunit C (Vma5p) of the yeast vacuolar H+-ATPase." J Biol Chem 277(11): 8979-8988.

De Milito, A. and S. Fais (2005). "Tumor acidity, chemoresistance and proton pump inhibitors." Future Oncol 1(6): 779-786. 
Di Cristofori, A., S. Ferrero, I. Bertolini, G. Gaudioso, M. V. Russo, V. Berno, M. Vanini, M. Locatelli, M. Zavanone, P. Rampini, T. Vaccari, M. Caroli and V. Vaira (2015). "The vacuolar H+ ATPase is a novel therapeutic target for glioblastoma." Oncotarget 6(19): 17514-17531.

Diboun, I., L. Wernisch, C. A. Orengo and M. Koltzenburg (2006). "Microarray analysis after RNA amplification can detect pronounced differences in gene expression using limma." BMC Genomics 7: 252.

Eisenberg, D., R. Luthy and J. U. Bowie (1997). "VERIFY3D: assessment of protein models with three-dimensional profiles." Methods Enzymol 277: 396-404.

Faronato, M., V. T. Nguyen, D. K. Patten, Y. Lombardo, J. H. Steel, N. Patel, L. Woodley, S. Shousha, G. Pruneri, R. C. Coombes and L. Magnani (2015). "DMXL2 drives epithelial to mesenchymal transition in hormonal therapy resistant breast cancer through Notch hyperactivation." Oncotarget 6(26): 22467-22479.

Feng, S., G. Zhu, M. McConnell, L. Deng, Q. Zhao, M. Wu, Q. Zhou, J. Wang, J. Qi, Y. P. Li and W. Chen (2013). "Silencing of atp6v1c1 prevents breast cancer growth and bone metastasis." Int J Biol Sci 9(8): 853-862.

Ferlay, J., M. Colombet, I. Soerjomataram, C. Mathers, D. M. Parkin, M. Pineros, A. Znaor and F. Bray (2018). "Estimating the global cancer incidence and mortality in 2018: GLOBOCAN sources and methods." Int J Cancer.

Forgac, M. (2007). "Vacuolar ATPases: rotary proton pumps in physiology and pathophysiology." Nat Rev Mol Cell Biol 8(11): 917-929.

Gao, J., B. A. Aksoy, U. Dogrusoz, G. Dresdner, B. Gross, S. O. Sumer, Y. Sun, A. Jacobsen, R. Sinha, E. Larsson, E. Cerami, C. Sander and N. Schultz (2013). "Integrative analysis of complex cancer genomics and clinical profiles using the cBioPortal." Sci Signal 6(269): pl1.

Hanahan, D. and R. A. Weinberg (2011). "Hallmarks of cancer: the next generation." Cell 144(5): 646-674.

Holliday, L. S. (2014). "Vacuolar H+-ATPase: An Essential Multitasking Enzyme in Physiology and Pathophysiology." New Journal of Science 2014: 1-21.

Holliday, L. S., M. Lu, B. S. Lee, R. D. Nelson, S. Solivan, L. Zhang and S. L. Gluck (2000). "The amino-terminal domain of the B subunit of vacuolar H+-ATPase contains a filamentous actin binding site." J Biol Chem 275(41): 32331-32337.

Ibrahim, S. A., A. Kulshrestha, G. K. Katara, M. A. Amin and K. D. Beaman (2016). "Cancer derived peptide of vacuolar ATPase 'a2' isoform promotes neutrophil migration by autocrine secretion of IL-8." Sci Rep 6: 36865.

Kallberg, M., H. Wang, S. Wang, J. Peng, Z. Wang, H. Lu and J. Xu (2012). "Template-based protein structure modeling using the RaptorX web server." Nat Protoc 7(8): 1511-1522.

Kane, P. M. and K. J. Parra (2000). "Assembly and regulation of the yeast vacuolar H(+)-ATPase." JExp Biol 203(Pt 1): 81-87.

Katara, G. K., M. K. Jaiswal, A. Kulshrestha, B. Kolli, A. Gilman-Sachs and K. D. Beaman (2014). "Tumor-associated vacuolar ATPase subunit promotes tumorigenic characteristics in macrophages." Oncogene 33(49): 5649-5654.

Kawasaki-Nishi, S., K. Bowers, T. Nishi, M. Forgac and T. H. Stevens (2001). "The amino-terminal domain of the vacuolar proton-translocating ATPase a subunit controls targeting and in vivo dissociation, and the carboxyl-terminal domain affects coupling of proton transport and ATP hydrolysis." J Biol Chem 276(50): 47411-47420.

Kawasaki-Nishi, S., T. Nishi and M. Forgac (2001). "Yeast V-ATPase complexes containing different isoforms of the 100-kDa a-subunit differ in coupling efficiency and in vivo dissociation." J Biol Chem 276(21): 17941-17948.

Kulshrestha, A., G. K. Katara, S. Ibrahim, S. Pamarthy, M. K. Jaiswal, A. Gilman Sachs and K. D. Beaman (2015). "Vacuolar ATPase 'a2' isoform exhibits distinct cell surface accumulation and modulates matrix metalloproteinase activity in ovarian cancer." Oncotarget 6(6): 3797-3810. 
Liao, C., B. Hu, M. J. Arno and B. Panaretou (2007). "Genomic screening in vivo reveals the role played by vacuolar $\mathrm{H}+$ ATPase and cytosolic acidification in sensitivity to DNA-damaging agents such as cisplatin." Mol Pharmacol 71(2): 416-425.

Liegeois, S., A. Benedetto, J. M. Garnier, Y. Schwab and M. Labouesse (2006). "The V0-ATPase mediates apical secretion of exosomes containing Hedgehog-related proteins in Caenorhabditis elegans." J Cell Biol 173(6): 949-961.

Lim, J. H., J. W. Park, S. J. Kim, M. S. Kim, S. K. Park, R. S. Johnson and Y. S. Chun (2007). "ATP6VOC competes with von Hippel-Lindau protein in hypoxia-inducible factor 1alpha (HIF-1alpha) binding and mediates HIF-1alpha expression by bafilomycin A1." Mol Pharmacol 71(3): 942-948. Lovell, S. C., I. W. Davis, W. B. Arendall, 3rd, P. I. de Bakker, J. M. Word, M. G. Prisant, J. S. Richardson and D. C. Richardson (2003). "Structure validation by Calpha geometry: phi,psi and Cbeta deviation." Proteins 50(3): 437-450.

Lu, Q., S. Lu, L. Huang, T. Wang, Y. Wan, C. X. Zhou, C. Zhang, Z. Zhang and X. Li (2013). "The expression of V-ATPase is associated with drug resistance and pathology of non-small-cell lung cancer." Diagn Pathol 8: 145.

Malhotra, S., O. K. Mathew and R. Sowdhamini (2015). "DOCKSCORE: a webserver for ranking protein-protein docked poses." BMC Bioinformatics 16: 127.

Manavalan, B. and J. Lee (2017). "SVMQA: support-vector-machine-based protein single-model quality assessment." Bioinformatics 33(16): 2496-2503.

Marchler-Bauer, A., Y. Bo, L. Han, J. He, C. J. Lanczycki, S. Lu, F. Chitsaz, M. K. Derbyshire, R. C. Geer, N. R. Gonzales, M. Gwadz, D. I. Hurwitz, F. Lu, G. H. Marchler, J. S. Song, N. Thanki, Z. Wang, R. A. Yamashita, D. Zhang, C. Zheng, L. Y. Geer and S. H. Bryant (2017). "CDD/SPARCLE: functional classification of proteins via subfamily domain architectures." Nucleic Acids Res 45(D1): D200D203.

Marshansky, V., J. L. Rubinstein and G. Gruber (2014). "Eukaryotic V-ATPase: novel structural findings and functional insights." Biochim Biophys Acta 1837(6): 857-879.

Martinez-Zaguilan, R., N. Raghunand, R. M. Lynch, W. Bellamy, G. M. Martinez, B. Rojas, D. Smith, W. S. Dalton and R. J. Gillies (1999). "pH and drug resistance. I. Functional expression of plasmalemmal V-type H+-ATPase in drug-resistant human breast carcinoma cell lines." Biochem Pharmacol 57(9): 1037-1046.

McConnell, M., S. Feng, W. Chen, G. Zhu, D. Shen, S. Ponnazhagan, L. Deng and Y. P. Li (2017). "Osteoclast proton pump regulator Atp6v1c1 enhances breast cancer growth by activating the mTORC1 pathway and bone metastasis by increasing V-ATPase activity." Oncotarget 8(29): 47675-47690.

Moal, I. H., B. Jimenez-Garcia and J. Fernandez-Recio (2015). "CCharPPI web server: computational characterization of protein-protein interactions from structure." Bioinformatics 31(1): 123-125.

Morel, N., J. C. Dedieu and J. M. Philippe (2003). "Specific sorting of the a1 isoform of the V$\mathrm{H}+\mathrm{ATPase}$ a subunit to nerve terminals where it associates with both synaptic vesicles and the presynaptic plasma membrane." J Cell Sci 116(Pt 23): 4751-4762.

Nancarrow, D. J., A. D. Clouston, B. M. Smithers, D. C. Gotley, P. A. Drew, D. I. Watson, S. Tyagi, N. K. Hayward, D. C. Whiteman, S. Australian Cancer and H. Study of Digestive (2011). "Whole genome expression array profiling highlights differences in mucosal defense genes in Barrett's esophagus and esophageal adenocarcinoma." PLoS One 6(7): e22513.

Nicolau-Neto, P., N. M. Da Costa, P. T. de Souza Santos, I. M. Gonzaga, M. A. Ferreira, S. Guaraldi, M. A. Moreira, H. N. Seuanez, L. Brewer, A. Bergmann, M. Boroni, A. L. Mencalha, C. D. P. Kruel, S. C. S. Lima, D. Esposito, T. A. Simao and L. F. R. Pinto (2018). "Esophageal squamous cell carcinoma transcriptome reveals the effect of FOXM1 on patient outcome through novel PIK3R3 mediated activation of PI3K signaling pathway." Oncotarget 9(24): 16634-16647.

Nishi, T. and M. Forgac (2002). "The vacuolar ( $\mathrm{H}+$ )-ATPases--nature's most versatile proton pumps." Nat Rev Mol Cell Biol 3(2): 94-103. 
Nishisho, T., K. Hata, M. Nakanishi, Y. Morita, G. H. Sun-Wada, Y. Wada, N. Yasui and T. Yoneda (2011). "The a3 isoform vacuolar type $\mathrm{H}(+)$-ATPase promotes distant metastasis in the mouse B16 melanoma cells." Mol Cancer Res 9(7): 845-855.

Olechnovic, K. and C. Venclovas (2017). "VoroMQA: Assessment of protein structure quality using interatomic contact areas." Proteins 85(6): 1131-1145.

Otero-Rey, E. M., M. Somoza-Martin, F. Barros-Angueira and A. Garcia-Garcia (2008). "Intracellular pH regulation in oral squamous cell carcinoma is mediated by increased V-ATPase activity via over-expression of the ATP6V1C1 gene." Oral Oncol 44(2): 193-199.

Pamarthy, S., A. Kulshrestha, G. K. Katara and K. D. Beaman (2018). "The curious case of vacuolar ATPase: regulation of signaling pathways." Mol Cancer 17(1): 41.

Perez-Sayans, M., M. D. Reboiras-Lopez, J. M. Somoza-Martin, F. Barros-Angueira, P. G. Diz, J. M. Rey and A. Garcia-Garcia (2010). "Measurement of ATP6V1C1 expression in brush cytology samples as a diagnostic and prognostic marker in oral squamous cell carcinoma." Cancer Biol Ther 9(12): 1057-1064.

Pettersen, E. F., T. D. Goddard, C. C. Huang, G. S. Couch, D. M. Greenblatt, E. C. Meng and T. E. Ferrin (2004). "UCSF Chimera--a visualization system for exploratory research and analysis." J Comput Chem 25(13): 1605-1612.

Puopolo, K., M. Sczekan, R. Magner and M. Forgac (1992). "The 40-kDa subunit enhances but is not required for activity of the coated vesicle proton pump." J Biol Chem 267(8): 5171-5176.

Rhodes, D. R., J. Yu, K. Shanker, N. Deshpande, R. Varambally, D. Ghosh, T. Barrette, A. Pandey and A. M. Chinnaiyan (2004). "ONCOMINE: a cancer microarray database and integrated datamining platform." Neoplasia 6(1): 1-6.

Ritchie, M. E., B. Phipson, D. Wu, Y. Hu, C. W. Law, W. Shi and G. K. Smyth (2015). "limma powers differential expression analyses for RNA-sequencing and microarray studies." Nucleic Acids Res 43(7): e47.

Roshandel, G., A. Nourouzi, A. Pourshams, S. Semnani, S. Merat and M. Khoshnia (2013). "Endoscopic screening for esophageal squamous cell carcinoma." Arch Iran Med 16(6): 351-357. Rustgi, A. K. and H. B. El-Serag (2014). "Esophageal carcinoma." N Engl J Med 371(26): 24992509.

Sali, A. and T. L. Blundell (1993). "Comparative protein modelling by satisfaction of spatial restraints." J Mol Biol 234(3): 779-815.

Samarao, S. S., C. E. Teodoro, F. E. Silva, C. C. Ribeiro, T. M. Granato, N. R. Bernardes, C. A. Retamal, A. R. Facanha, A. L. Okorokova-Facanha and L. A. Okorokov (2009). "V H+-ATPase along the yeast secretory pathway: energization of the ER and Golgi membranes." Biochim Biophys Acta 1788(2): 303-313.

Sennoune, S. R., K. Bakunts, G. M. Martinez, J. L. Chua-Tuan, Y. Kebir, M. N. Attaya and R. Martinez-Zaguilan (2004). "Vacuolar H+-ATPase in human breast cancer cells with distinct metastatic potential: distribution and functional activity." Am J Physiol Cell Physiol 286(6): C1443-1452.

Sennoune, S. R. and R. Martinez-Zaguilan (2012). "Vacuolar H(+)-ATPase signaling pathway in cancer." Curr Protein Pept Sci 13(2): 152-163.

Serra-Peinado, C., A. Sicart, J. Llopis and G. Egea (2016). "Actin Filaments Are Involved in the Coupling of V0-V1 Domains of Vacuolar H+-ATPase at the Golgi Complex." J Biol Chem 291(14): 7286-7299.

Smardon, A. M. and P. M. Kane (2007). "RAVE is essential for the efficient assembly of the C subunit with the vacuolar H(+)-ATPase." J Biol Chem 282(36): 26185-26194.

Smith, A. N., K. J. Borthwick and F. E. Karet (2002). "Molecular cloning and characterization of novel tissue-specific isoforms of the human vacuolar $\mathrm{H}(+)$-ATPase $\mathrm{C}, \mathrm{G}$ and d subunits, and their evaluation in autosomal recessive distal renal tubular acidosis." Gene 297(1-2): 169-177.

Son, S. W., S. H. Kim, E. Y. Moon, D. H. Kim, S. Pyo and S. H. Um (2016). "Prognostic significance and function of the vacuolar H+-ATPase subunit V1E1 in esophageal squamous cell carcinoma." Oncotarget 7(31): 49334-49348. 
Stransky, L., K. Cotter and M. Forgac (2016). "The Function of V-ATPases in Cancer." Physiol Rev 96(3): 1071-1091.

Strasser, B., J. Iwaszkiewicz, O. Michielin and A. Mayer (2011). "The V-ATPase proteolipid cylinder promotes the lipid-mixing stage of SNARE-dependent fusion of yeast vacuoles." EMBO J 30(20): 4126-4141.

Sukhwal, A. and R. Sowdhamini (2015). "PPCheck: A Webserver for the Quantitative Analysis of Protein-Protein Interfaces and Prediction of Residue Hotspots." Bioinform Biol Insights 9: 141151.

Sun-Wada, G. H., Y. Murata, M. Namba, A. Yamamoto, Y. Wada and M. Futai (2003). "Mouse proton pump ATPase $\mathrm{C}$ subunit isoforms (C2-a and $\mathrm{C} 2-\mathrm{b}$ ) specifically expressed in kidney and lung." J Biol Chem 278(45): 44843-44851.

Sun-Wada, G. H., T. Yoshimizu, Y. Imai-Senga, Y. Wada and M. Futai (2003). "Diversity of mouse proton-translocating ATPase: presence of multiple isoforms of the $C, d$ and $G$ subunits." Gene 302(1-2): 147-153.

Toei, M., R. Saum and M. Forgac (2010). "Regulation and isoform function of the V-ATPases." Biochemistry 49(23): 4715-4723.

Toyomura, T., Y. Murata, A. Yamamoto, T. Oka, G. H. Sun-Wada, Y. Wada and M. Futai (2003). "From lysosomes to the plasma membrane: localization of vacuolar-type H+-ATPase with the a3 isoform during osteoclast differentiation." J Biol Chem 278(24): 22023-22030.

Uziela, K., D. Menendez Hurtado, N. Shu, B. Wallner and A. Elofsson (2017). "ProQ3D: improved model quality assessments using deep learning." Bioinformatics 33(10): 1578-1580.

van Zundert, G. C. P., J. Rodrigues, M. Trellet, C. Schmitz, P. L. Kastritis, E. Karaca, A. S. J. Melquiond, M. van Dijk, S. J. de Vries and A. Bonvin (2016). "The HADDOCK2.2 Web Server: UserFriendly Integrative Modeling of Biomolecular Complexes." J Mol Biol 428(4): 720-725.

Vangone, A. and A. M. Bonvin (2015). "Contacts-based prediction of binding affinity in proteinprotein complexes." Elife 4: e07454.

Vitavska, O., H. Merzendorfer and H. Wieczorek (2005). "The V-ATPase subunit C binds to polymeric F-actin as well as to monomeric $\mathrm{G}$-actin and induces cross-linking of actin filaments." J Biol Chem 280(2): 1070-1076.

Voss, M., O. Vitavska, B. Walz, H. Wieczorek and O. Baumann (2007). "Stimulus-induced phosphorylation of vacuolar H(+)-ATPase by protein kinase A." J Biol Chem 282(46): 3373533742.

Wagner, C. A., K. E. Finberg, S. Breton, V. Marshansky, D. Brown and J. P. Geibel (2004). "Renal vacuolar H+-ATPase." Physiol Rev 84(4): 1263-1314.

Webb, B. A., M. Chimenti, M. P. Jacobson and D. L. Barber (2011). "Dysregulated pH: a perfect storm for cancer progression." Nat Rev Cancer 11(9): 671-677.

Whitton, B., H. Okamoto, G. Packham and S. J. Crabb (2018). "Vacuolar ATPase as a potential therapeutic target and mediator of treatment resistance in cancer." Cancer Med 7(8): 38003811.

Wiederstein, M. and M. J. Sippl (2007). "ProSA-web: interactive web service for the recognition of errors in three-dimensional structures of proteins." Nucleic Acids Res 35(Web Server issue): W407-410.

Wojtkowiak, J. W., D. Verduzco, K. J. Schramm and R. J. Gillies (2011). "Drug resistance and cellular adaptation to tumor acidic pH microenvironment." Mol Pharm 8(6): 2032-2038.

$\mathrm{XU}, \mathrm{D}$. and Y. Zhang (2011). "Improving the physical realism and structural accuracy of protein models by a two-step atomic-level energy minimization." Biophys J 101(10): 2525-2534.

Xu, J., R. Xie, X. Liu, G. Wen, H. Jin, Z. Yu, Y. Jiang, Z. Zhao, Y. Yang, B. Ji, H. Dong and B. Tuo (2012). "Expression and functional role of vacuolar $\mathrm{H}(+)$-ATPase in human hepatocellular carcinoma." Carcinogenesis 33(12): 2432-2440.

Xue, L. C., J. P. Rodrigues, P. L. Kastritis, A. M. Bonvin and A. Vangone (2016). "PRODIGY: a web server for predicting the binding affinity of protein-protein complexes." Bioinformatics 32(23): 3676-3678. 
Yang, J., R. Yan, A. Roy, D. Xu, J. Poisson and Y. Zhang (2015). "The I-TASSER Suite: protein structure and function prediction." Nat Methods 12(1): 7-8.

Zoncu, R., L. Bar-Peled, A. Efeyan, S. Wang, Y. Sancak and D. M. Sabatini (2011). "mTORC1 senses lysosomal amino acids through an inside-out mechanism that requires the vacuolar $\mathrm{H}(+)-$ ATPase." Science 334(6056): 678-683.

Zuo, J., S. Vergara, S. Kohno and L. S. Holliday (2008). "Biochemical and functional characterization of the actin-binding activity of the B subunit of yeast vacuolar H+-ATPase." J Exp Biol 211(Pt 7): 1102-1108.

\section{SUPPLEMENTARY MATERIALS}

Figure S1. Expression levels of $A$ and $B$ subunits of V-ATPase in tumor, normalappearing surrounding tissue, and healthy esophageal mucosa.

Figure S2. Expression levels of $c$ " subunit and $d$ and $G$ isoforms in tumor, normalappearing surrounding tissue and healthy esophageal mucosa.

Figure S3. Expression levels of a3 and a4 isoforms in tumor, normal-appearing surrounding tissue and healthy esophageal mucosa.

Figure S4. mRNA expression pattern of V-ATPase genes in several cancer types.

FigureS5. Distinctive balance of $\mathrm{C}$ isoforms.

FigureS6. $C$ isoforms mRNA expression in esophageal cancer histological subtypes.

Figure S7. Protein-protein dockings between proposed hC2a models with already resolved structure of PKC and PKA proteins.

Figure S8. Putative actin-binding sites in $\mathrm{C}$ isoforms.

Table 1. Fold-change mean values comparing ESCC and normal surrounding tissues for each $V$-ATPase gene.

Table 2. Similarities between V-ATPase $C$ subunits.

Table 3. Serine kinase/phosphatase motifs present in the additional exon of hC2a isoform.

Table 4. Phosphorylated serine-based motifs in the additional exon of hC2a isoform.

Table 5. Characteristics of the individuals included in the study.

Table 6. Oligonucleotide sequences and amplicon size. 